\title{
Structure and stability of self-assembled actin-lysozyme complexes in salty water
}

\author{
Lori K. Sanders, ${ }^{1}$ Camilo Guáqueta, ${ }^{1}$ Thomas E. Angelini, ${ }^{2}$ Jae-Wook Lee, ${ }^{1}$ \\ Scott C. Slimmer, ${ }^{1}$ Erik Luijten,, , and Gerard C. L. Wong ${ }^{1,2, \boldsymbol{\theta}}$ \\ ${ }^{1}$ Dept. of Materials Science and Engineering, University of Illinois at Urbana-Champaign, Urbana, Illinois 61801 \\ ${ }^{2}$ Dept. of Physics, University of Illinois at Urbana-Champaign, Urbana, Illinois 61801
}

(Dated: September 15, 2021)

\begin{abstract}
Interactions between actin, an anionic polyelectrolyte, and lysozyme, a cationic globular protein, have been examined using a combination of synchrotron small-angle x-ray scattering and molecular dynamics simulations. Lysozyme initially bridges pairs of actin filaments, which relax into hexagonally-coordinated columnar complexes comprised of actin held together by incommensurate one-dimensional close-packed arrays of lysozyme macroions. These complexes are found to be stable even in the presence of significant concentrations of monovalent salt, which is quantitatively explained from a redistribution of salt between the condensed and the aqueous phases.
\end{abstract}

PACS numbers: 82.35.Rs, 87.16.Ka, 87.64.Bx, 87.15.Aa

In the presence of multivalent cations, anionic biological polyelectrolytes can overcome their electrostatic repulsion and exhibit a mutual attraction. These "likecharge attractions" result from ion correlations that cannot be understood within mean-field theories such as the commonly-employed Poisson-Boltzmann formalism [1, 2, 3]. The problem becomes more complex when the mediating multivalent cations are themselves macroions. Macroion-polyelectrolyte complexes occur in many physical systems, such as DNA-dendrimer complexes for nonviral gene therapy [4] and antimicrobial binding in cystic fibrosis [5]. Various factors affect their formation: The presence of salt can lead to an attraction driven by osmotic pressure [6]. Differential screening of positive and negative charges distributed on the surface of a macroion may significantly modify interactions at the macroion-polyelectrolyte interface [7]. Entropic gain due to mutual neutralization and consequent counterion release upon macroion-polyelectrolyte "adhesion" is expected to be important, but can be potentially modulated by the steric commensurability between the charge pattern on the polyelectrolyte and the macroion size [8]. The relative importance of all these interactions, and how they modify one another in their combined effect on the structural evolution of macroion-polyelectrolyte complexes, is generally unknown.

In this Letter, we examine the role of several of the above-mentioned interactions in the complexation of actin and lysozyme, a prototypical system of oppositelycharged "rods" and "spheres," over a range of monovalent salt concentrations. Using synchrotron small-angle $\mathrm{x}$-ray scattering (SAXS), we show that self-assembled complexes are comprised of hexagonally-coordinated columnar arrangements of actin held together by onedimensional (1D) arrays of lysozyme macroions at the three-fold interstitial "tunnels" of the columnar actin sublattice (Fig. 1). Molecular dynamics (MD) simulations using a realistic model of the actin helix provide a detailed confirmation of this picture, and reveal
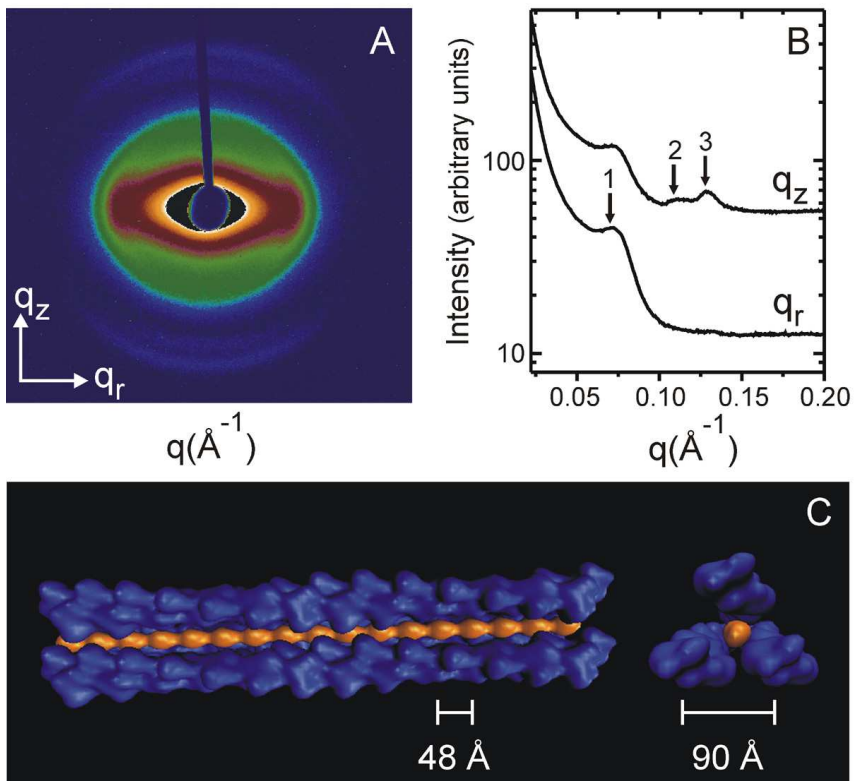

FIG. 1: (A) Synchrotron 2D x-ray diffraction pattern of partially aligned actin-lysozyme bundles, formed in a solution containing $150 \mathrm{mM} \mathrm{KCl}$. (B) $1 \mathrm{D}$ integrated slices along the $q_{z}$ and $q_{r}$ directions with arrows marking the actin-actin closepacked bundling peak (1), the actin helix form factor (2), and the lysozyme-lysozyme correlation peak (3). (C) Proposed structure of actin-lysozyme composite bundles (side and end views): Lysozyme (orange) is close-packed in three-fold symmetric sites between actin filaments (blue).

structural reconstructions and corresponding salt redistribution within an actin-lysozyme bundle as the interactin separation is varied. Both experiment and simulation show that the lysozyme is arranged in a closepacked manner, incommensurate with the actin periodicity. Moreover, the self-assembly of columnar actinlysozyme complexes is enhanced for higher concentrations of monovalent ions. We believe that these results can be explained by significant repartitioning of salt 
between the condensed and the aqueous phases, which strongly modifies screening effects.

$\mathrm{F}$-actin is an anionic rod-like cytoskeletal polymer (diameter $7.5 \mathrm{~nm}$, charge density $-e / 0.25 \mathrm{~nm}$, persistence length $10 \mu \mathrm{m})$. Lysozyme is approximately an ellipsoid of size $2.6 \mathrm{~nm} \times 2.6 \mathrm{~nm} \times 4.5 \mathrm{~nm}$ with a net charge of $+9 e$ at neutral $\mathrm{pH}$. Monomeric G-actin (MW 42,000) was prepared from a lyophilized powder of rabbit skeletal muscle. The non-polymerizing G-actin solution contained a $5 \mathrm{mM}$ TRIS buffer at $\mathrm{pH} 8.0$, with $0.2 \mathrm{mM} \mathrm{CaCl}_{2}$, $0.5 \mathrm{mM}$ ATP, and $0.2 \mathrm{mM}$ DTT and $0.01 \% \mathrm{NaN}_{3}$. Gactin $(2 \mathrm{mg} / \mathrm{ml})$ was polymerized into F-actin upon the addition of salt $(100 \mathrm{mM} \mathrm{KCl})$. Human plasma gelsolin was used to control the average F-actin length to $\sim 1 \mathrm{~mm}$. The F-actin filaments were treated with phalloidin (MW 789.2) to prevent depolymerization. Hen egg white lysozyme (MW 14,300) was mixed with F-actin in $1.5 \mathrm{~mm}$ diameter quartz capillaries to form isoelectric actin-lysozyme complexes. SAXS experiments were performed both at Beamline 4-2 of the Stanford Synchrotron Radiation Laboratory as well as at an in-house $\mathrm{x}$-ray source. The incident synchrotron $x$-rays from the 8-pole Wiggler were monochromatized to $8.98 \mathrm{KeV}(\lambda=1.3806 \AA)$ using a double-bounce $\mathrm{Si}(111)$ crystal, focused using a cylindrical mirror. The scattered radiation was collected using an MAR Research charge-coupled device camera (pixel size $79 \mu \mathrm{m}$ ). For the in-house experiments, incident $\mathrm{CuK}_{\alpha}$ radiation $(\lambda=1.54 \AA)$ from a Rigaku rotatinganode generator was monochromatized and focused using Osmic confocal multilayer optics, and scattered radiation was collected on a Bruker 2D wire detector (pixel size $105 \mu \mathrm{m}$ ). The 2D SAXS data from both systems are mutually consistent.

A 2D diffraction pattern for partially aligned isoelectric F-actin-lysozyme bundles and its associated 1D integrated slices along the $q_{z}$ and $q_{r}$ directions are shown in Figs. 1A and 1B. Examination of the slice along the equatorial $\left(q_{r}\right)$ direction shows a correlation peak at $q=0.07 \AA^{-1}$ that corresponds to close-packed composite actin-lysozyme bundles. The inter-actin spacing of $90 \AA$ is consistent with a columnar actin lattice expanded by lysozyme in three-fold interstitial "tunnels," aligned with its long axis parallel to the actin. This inter-actin spacing is significantly larger than the $75 \AA$ spacing for close-packed actin condensed with multivalent ions 9 ]. No other arrangement of lysozyme and actin will reproduce this diffraction pattern, given their respective sizes. Along the meridional $\left(q_{z}\right)$ direction, a weak, mosaicsmeared actin form factor feature at $0.113 \AA^{-1}$ is observed, as well as a new, strong correlation peak that differs from expected actin form factor features (layer lines) in position, orientation, and relative intensity. The appearance of this peak at $q_{z}=0.130 \AA^{-1}$ corresponds to an inter-lysozyme distance of $48.3 \AA$, comparable to the length of lysozyme along its major axis, which suggests that lysozyme is close-packed along this direction within the bundles. Interestingly, this lysozyme periodicity is incommensurate with the projected actin periodicity $(\sim 56 \AA)$, in contrast with the behavior of divalent ions on actin [9]. This incommensurate arrangement permits charge matching between actin and lysozyme within the bundle, and indicates the important role of entropy gain from counterion release in this system. Figure $1 \mathrm{C}$ shows schematic representations of a condensed bundle.

In order to elucidate the underlying mechanism for bundle formation and the structure of the resulting complex, we have performed MD simulations using a modified version of Moldy [10]. In these simulations, Gactin is modeled using the four-sphere model [1], which is based upon crystallographic measurements and provides a relatively accurate coarse-grained representation of the monomer charge distribution. F-actin is comprised of a sequence of these monomers, in which successive units have a separation of $27.5 \AA$ and a relative rotation of $166.7^{\circ}$ around the filament axis. This leads to a helical structure with a repeat unit of 13 monomers. The filaments are assembled into a parallel hexagonallycoordinated bundle. An elementary simulation cell consists of a bundle fragment containing $2 \times 2$ filaments with a length of 6 repeat units ( 78 monomers) each. This cell is periodically replicated in all directions. Following the experiments, we set the lysozyme concentration in the bundle to neutralizing conditions, which corresponds to 352 lysozyme units per simulation cell. Each lysozyme is modeled as a rigid dumbbell structure of two spheres with diameter $25 \AA$ and charge $4.5 e$, at a center-to-center distance of $20 \AA$, thus approximating the aforementioned ellipsoidal dimensions. Additional salt is modeled as monovalent spherical particles with a hydrated radius of $3.3 \AA$. Coulomb interactions are treated by means of Ewald summation, and excluded-volume interactions are

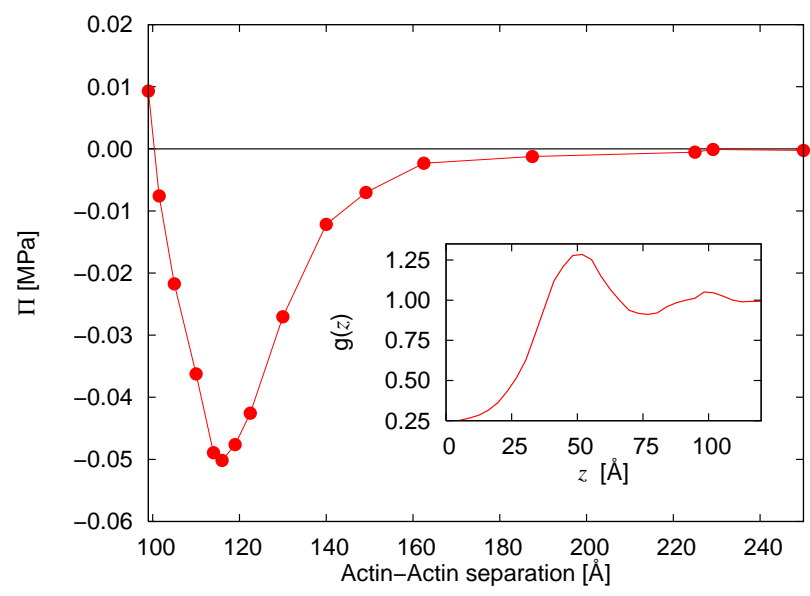

FIG. 2: Osmotic pressure of a hexagonally-coordinated bundle of actin filaments in excess solution, as determined from MD simulations. Inset: lysozyme pair correlation function along the filament axis. For discussion see the text. 
represented by pair potentials of the form $k_{\mathrm{B}} T(\sigma / r)^{12}$, where $\sigma$ is the sum of the effective radii of two interacting particles (ions, G-actin subunits and lysozyme subunits). During each simulation, the actin separation is fixed, whereas lysozyme and all ions move freely. Thus, the simulations probe the stability of a swelling bundle while maintaining the filaments in a parallel configuration, ignoring their rotational degrees of freedom. This is justified by the observation that the calculations are confined to actin separations below $25 \mathrm{~nm}$, i.e., less than $0.25 \%$ of the persistence length. Mutual sliding and axial rotation of the filaments are not taken into account.

This model indeed predicts electrostatically driven complex formation. Since the water is modeled as a dielectric continuum, the osmotic pressure $\Pi$ can be obtained directly from the virial involving all interparticle forces 12. Bundle formation takes place in excess solution, and hence the bundle stability follows from a comparison of the osmotic pressure to the osmotic pressure of the salt $\Pi_{\text {salt }}$. A negative osmotic pressure difference $\Delta \Pi \equiv \Pi-\Pi_{\text {salt }}$ implies bundle contraction and the freeenergy minimum $[\Delta \Pi=0$ and $\partial(\Delta \Pi) / \partial V<0]$ yields the stable actin separation. A comparable approach has been employed before (see Refs. 13, 14] and references therein) to study the condensation of rod-like polyelectrolytes by counterions. Figure 2 shows that, under saltfree conditions, an inter-actin spacing of $\sim 100 \AA$ is predicted, in quite close agreement with the experimental observations.

Having established that our model captures essential aspects of actin-lysozyme complexation, we exploit it to elucidate the structural properties and evolution of the resulting complexes. Figure 3 shows contour plots of the lysozyme center of mass, projected on a plane perpendicular to the bundle axis. In order to minimize artifacts resulting from the finite actin length, the calculations employed filaments consisting of 12 repeat units (156 monomers). In the equilibrium configuration (Fig. 3A), the maximum lysozyme concentration occurs in the three-fold interstitial regions between the actin filaments, supporting the interpretation of the SAXS diffraction data in Fig. [1 Furthermore, the lysozyme pair correlation function (inset of Fig. 2) along the actin axis shows a clear peak at $z=50 \AA$, corroborating the experimentally measured close-packed value of $48.3 \AA$ 15]. Minor enhancements of the lysozyme concentration can also be seen in the bridging regions between pairs of neighboring filaments. The lysozyme distribution is governed both by entropic effects and by a competition between electrostatic protein repulsions and actin-lysozyme attractions. Also, the helical actin structure imposes an excluded-volume repulsion with a rather pronounced shape. In order to distinguish any structure resulting from this repulsion, Fig. 3 B shows a contour plot obtained in a simulation without electrostatic effects. The homogeneous lysozyme distribution confirms that the

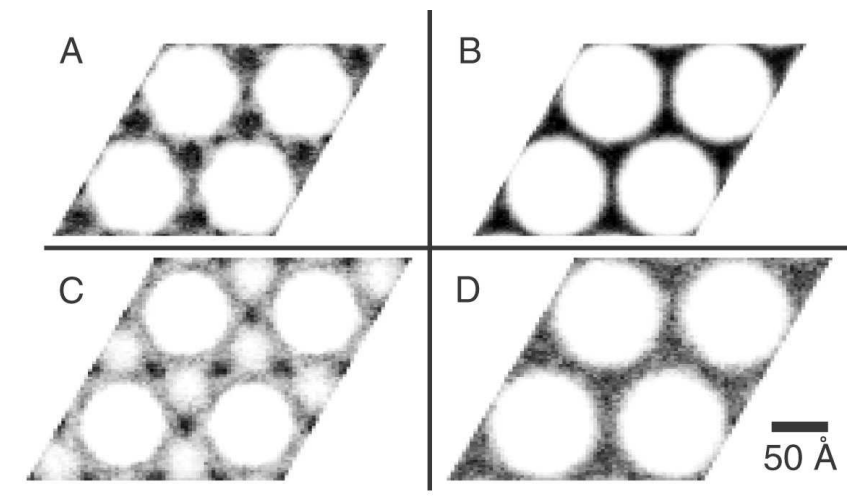

FIG. 3: Contour plots showing the lysozyme distribution in actin-lysozyme complexes without added salt. Darker shading corresponds to higher concentrations. In the equilibrium configuration (A), lysozyme is predominantly located in the three-fold interstitial regions. The free-energy inflection point occurs at a slightly expanded lattice (C), in which lysozyme is depleted from these regions and occupies the bridging regions instead. Panels (B) and (D) are the counterparts of panels (A) and $(\mathrm{C})$, respectively, in the absence of electrostatic forces.

structure in Fig. 3A is dominated by electrostatic interactions. Interestingly, the actin excluded volume (white circular regions) also has a more pronounced hexagonal structure in panel $\mathrm{A}$, which is caused by the precessing highly-charged regions on the monomers. Consideration of larger lattice spacings provides information on intermediate states that may arise during the complexation process. As illustrated in Fig. $3 \mathrm{C}$, significant rearrangements occur in the final stages of the bundle formation: At the osmotic-pressure minimum (free-energy inflection point), i.e., at an actin separation that is increased by merely $16 \AA$, lysozyme is depleted from the interstitial regions and instead predominantly occupies the bridging sites. Figure 3D confirms that this lysozyme distribution is again dominated by electrostatic effects. Thus, the complex evolves from lysozyme at bridging positions between pairs of actin rods to the final three-fold positions observed by experiment. Lysozyme and actin "contact" interactions are maintained during this structural relaxation, indicating that their mutual electrostatic attraction plays an important role.

It is interesting to consider how this self-assembly is affected by the addition of monovalent salt. The counterion release mechanism implicit in actin-lysozyme binding will strongly modify qualitative arguments based on screening. The repartitioning of salt between the condensed and aqueous phases implies a different degree of screening inside and outside the complex. In addition, it can lead to a stabilizing external osmotic pressure. Such a redistribution of ions is often not taken into account theoretically. To study these effects, a series of SAXS measurements were performed on actin-lysozyme complexes at different $\mathrm{NaCl}$ concentrations. As $[\mathrm{NaCl}]$ is 


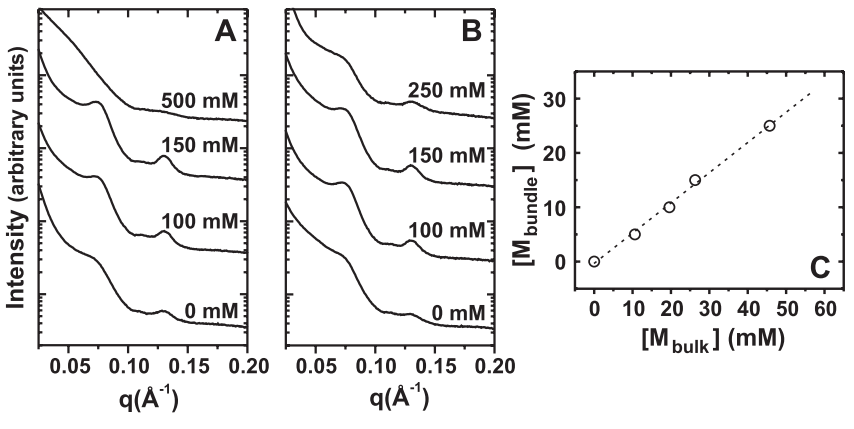

FIG. 4: Series of diffraction data showing the evolution of bundle structure as a function of (A) $\mathrm{NaCl}$ and (B) $\mathrm{KCl}$ concentration with maximum bundling occurring around $120 \mathrm{mM}$. (C) Simulated (o) bundle vs. bulk ion concentrations for a stabilized actin-lysozyme complex. Salt repartitions into different concentrations inside and outside the bundle. The dashed line is a guide to the eye.

increased to $150 \mathrm{mM}$, the turbidity increases and the intensity of the lysozyme-actin diffraction peak increases without significant changes in its peak width (Fig. 4A A), indicating the formation of more bundles (rather than bundles that are more ordered and have larger coherent domains). At higher salt concentrations, the trend reverses and a weakening of the bundling peak is observed. The same results are found using $\mathrm{KCl}$ (Fig. $4 \mathrm{~B}$ ), showing that this is not a cation-specific binding effect.

Since regular screening is likely to play a role in the ultimate disappearance of the bundle, we concentrate on the stability at low and intermediate salt levels. Simulations of bundles with additional salt show that the osmotic pressure within the complex rises more rapidly as a function of salt concentration than the bulk pressure of salt at the same concentration, leading to destabilization once the salt concentration exceeds $\sim 10 \mathrm{mM}$. This apparent discrepancy with the experimental findings already suggests a redistribution of salt ions. To quantify this further, grand-canonical simulations of the bundle can be employed [13]. Here, we have chosen an alternative strategy. Using the Widom particle-insertion technique [16], we determine the chemical potential of salt within the complex, as a function of concentration and actin separation. Exploiting the coexistence condition, we subsequently determine the corresponding bulk salt concentrations and osmotic pressures via independent grandcanonical Monte Carlo simulations. This yields several important results. Firstly, the salt concentration inside the actin-lysozyme complex is approximately twice lower than the bulk concentration, which leads to a difference in osmotic pressure that is sufficient to maintain bundle stability up to much higher concentrations than otherwise would have been possible (Fig. 4C). Secondly, the depression of the ion concentration within the bun- dle may explain why maximal actin-lysozyme bundling is observed at a global salt concentration around $120 \mathrm{mM}$, rather than at concentrations in the range $30-60 \mathrm{mM}$ as predicted from differential screening arguments [7].

In summary, by studying self-assembled actinlysozyme complexes via a combination of small-angle $\mathrm{x}$ ray scattering and molecular dynamics simulations, we have shown that salt repartitioning impinges strongly on the structure and stability of the complex, and qualifies commonly-invoked mechanisms such as counterion release and differential screening.

We gratefully acknowledge discussions with M. Olvera de la Cruz, P. Dubin, M. Rubinstein, and D. Harries. This material is based upon work supported by the U.S. Department of Energy, Division of Materials Sciences under Award No. DEFG02-91ER45439, through the Frederick Seitz Materials Research Laboratory at the University of Illinois at Urbana-Champaign and by the National Science Foundation under Grants DMR-0346914 (to EL), DMR-0409369 (to GW), and CTS-0120978.

* Corresponding author. E-mail: luijten@uiuc.edu

$\dagger$ Corresponding author. E-mail: gclwong@uiuc.edu

[1] W. M. Gelbart, R. F. Bruinsma, P. A. Pincus, and V. A. Parsegian, Physics Today 53, 38 (September 2000).

[2] A. Y. Grosberg, T. T. Nguyen, and B. I. Shklovskii, Rev. Mod. Phys. 74, 329 (2002).

[3] Y. Levin, Rep. Prog. Phys. 65, 1577 (2002).

[4] H. M. Evans et al., Phys. Rev. Lett. 91, 075501 (2003).

[5] D. J. Weiner, R. Bucki, and P. A. Janmey, Am. J. Respir. Cell Mol. Biol. 28, 738 (2003).

[6] V. A. Parsegian, R. P. Rand, and D. C. Rau, Methods Enzymol. 259, 43 (1995).

[7] E. Seyrek, P. L. Dubin, C. Tribet, and E. A. Gamble, Biomacromol. 4, 273 (2003).

[8] M. L. Henle, C. D. Santangelo, D. M. Patel, and P. A. Pincus, Europhys. Lett. 66, 284 (2004).

[9] T. E. Angelini, H. Liang, W. Wriggers, and G. C. L. Wong, Proc. Natl. Acad. Sci. U.S.A. 100, 8634 (2003).

[10] K. Refson, Comp. Phys. Comm. 126, 310 (2000).

[11] H. A. Al-Khayat, N. Yagi, and J. M. Squire, J. Mol. Biol. 252, 611 (1995).

[12] T. L. Hill, Statistical Mechanics: Principles and Selected Applications (McGraw-Hill, New York, 1956).

[13] A. P. Lyubartsev, J. X. Tang, P. A. Janmey, and L. Nordenskiöld, Phys. Rev. Lett. 81, 5465 (1998).

[14] M. Deserno, A. Arnold, and C. Holm, Macromolecules 36, 249 (2003).

[15] To avoid cross-correlations between different three-fold interstitial regions, the pair correlation function is computed for each region separately.

[16] D. Frenkel and B. Smit, Understanding Molecular Simulation, 2nd ed. (Academic, San Diego, 2002). 